Astronomy Letters, 2017, Vol. 43, No 8, pp. 559-566.

\title{
Searching for Stars Closely Encountering with the Solar System Based on Data from the Gaia DR1 and RAVE5 Catalogues
}

\author{
V.V. Bobylev 1 and A.T. Bajkova \\ Central (Pulkovo) Astronomical Observatory, Russian Academy of Sciences, \\ Pulkovskoe sh. 65, St. Petersburg, 196140 Russia
}

\begin{abstract}
We have searched for the stars that either encountered in the past or will encounter in the future with the Solar system closer than 2 pc. For this purpose, we took more than 216000 stars with the measured proper motions and trigonometric parallaxes from the Gaia DR1 catalogue and their radial velocities from the RAVE5 catalogue. We have found several stars for which encounters closer than $1 \mathrm{pc}$ are possible. The star GJ 710 , for which the minimum distance is $d_{m}=0.063 \pm 0.044 \mathrm{pc}$ at time $t_{m}=1385 \pm 52$ thousand years, is the record-holder among them. Two more stars, TYC 8088-631-1 and TYC 6528-980-1, whose encounter parameters, however, are estimated with large errors, are of interest.
\end{abstract}

DOI: $10.1134 / \mathrm{S} 1063773717080011$

\section{INTRODUCTION}

According to the hypothesis of Oort (1950), the Solar system is surrounded by a comet cloud. Although there are little reliable data on this cloud, it is highly likely that it has a spherical shape and a radius $1 \times 10^{5} \mathrm{AU}(0.49 \mathrm{pc})$. The total number of comets is supposed to be $10^{11}$. The flybys of Galactic field stars near the Oort cloud can trigger the formation of comet showers moving into the region of the major planets (Hills 1981). In the long run, the possibility that the Moon and the Earth are bombarded with such comets is not ruled out (Wickramasinghe and Napier 2008).

The long-term evolution of the Oort cloud was considered on the basis of numerical simulations, for example, in Emel'yanenko et al. (2007), Leto et al. (2008), Rickman et al. (2008), and Dybczyński and Królikowska (2011). In particular, the Jupiter-Saturn system was shown to be a tangible barrier leading to a redistribution of the density of comets in the cloud. Apart from the flybys of stars, the Oort cloud is perturbed by giant molecular clouds and the gravitational tide produced by the Galactic attraction (Dybczyński 2002, 2005; Martinez-Barbosa et al. 2017).

Matthews (1994), Mülläri and Orlov (1996), Garcia-Sánchez et al. (1999, 2001), Bobylev (2010a, 2010b), Anderson and Francis (2012), Dybczyński and Berski (2015), Bailer-Jones (2015), Feng and Bailer-Jones (2015), and Mamajek et al. (2015) searched for the close encounters of stars with the solar orbit using various observational data. As a result, $\sim 200$ Hipparcos (1997) stars that either encountered or would encounter with the Solar system

\footnotetext{
${ }^{1}$ e-mail: vbobylev@gao.spb.ru
} 
closer than 5 pc in the time interval from -10 to +10 Myr were revealed. Several candidates have a high probability of their penetration into the Oort cloud region.

For example, the star HIP 85605 (a dwarf of spectral type K4) may encounter with the Solar system within a distance $d_{m} \sim 0.1 \mathrm{pc}$ at $\mathrm{tm} \sim 330$ thousand years (Bailer-Jones 2015); for the star HIP $63721(\mathrm{~F} 3 \mathrm{~V})$ these parameters are $d_{m} \sim 0.2 \mathrm{pc}$ and $t_{m} \sim 150$ thousand years (Bailer-Jones 2015; Dybczyński and Berski 2015). At the same time, all authors point out that the parallaxes of the stars HIP 85605 and HIP 63721 are very unreliable.

The low-mass binary system WISE J072003.20-084651.2 (M9.5 + T5) with a total mass of $\sim 0.15 M_{\odot}$ is of interest. Mamajek et al. (2015) estimated $d_{m}=0.25_{-0.07}^{+0.11} \mathrm{pc}$ and $t_{m}=$ $-70_{-0.10}^{+0.15}$ thousand years for it.

The star GL 710 (K7V), for which the encounter parameters found from the Hipparcos data are $d_{m}=0.31 \pm 0.17 \mathrm{pc}$ and $t_{m}=1447 \pm 60$ thousand years (Garcia-Sánchez et al. 2001; Bobylev 2010a), is well known. Completely new estimates of these parameters have recently been obtained by Berski and Dybczyński (2016) using the parallaxes and proper motions measured in the Gaia experiment (Prusti et al. 2016): $d_{m}=0.065 \pm 0.030 \mathrm{pc}$ and $t_{m}=1350 \pm 50$ thousand years. Thus, the star GJ 710 remains the record-holder in terms of encounters among the candidate stars with more or less reliable measurements.

New possibilities in searching for stars closely encountering with the Solar system are associated with the appearance of the first version of the Gaia catalogue. This catalogue was produced from a combination of the data in the first year of Gaia observations with the positions and proper motions of Tycho-2 stars (Hog et al. 2000). It is designated as TGAS (Tycho-Gaia Astrometric Solution, Michalik et al. 2015; Brown et al. 2016; Lindegren et al. 2016) and contains the parallaxes and proper motions of $\sim 2$ million bright stars. The TGAS version has no stellar radial velocities; therefore, specialized RAVE type catalogues of radial velocities should be invoked to calculate the total space velocities of stars.

The goal of this paper is the search for candidate stars closely encountering with the Sun based on the present-day data on stars. For this purpose, we use the Gaia DR1 and RAVE5 catalogues (Kunder et al. 2017). We construct the orbits of stars using an improved model Galactic gravitational potential (Bajkova and Bobylev 2016).

\section{DATA}

The random errors of the parameters included in the Gaia DR1 catalogue are either comparable to or smaller than those given in the Hipparcos and Tycho- 2 catalogues. The mean parallax errors are $\sim 0.3$ mas (milliarcseconds). For most stars of the TGAS version the mean

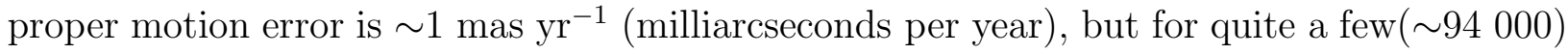
stars common to the Hipparcos catalogue this error is smaller by an order of magnitude, 0.06 mas $\mathrm{yr}^{-1}$.

The RAVE (RAdial Velocity Experiment) project (Steinmetz et al. 2006) is devoted to determining the radial velocities of many faint stars. The observations in the southern hemisphere at the 1.2-m Schmidt telescope of the Anglo-Australian Observatory started in 2003. Five data releases of this catalogue (DR1-DR5) have been published since then. The mean radial velocity error is $\sim 3 \mathrm{~km} \mathrm{~s}^{-1}$. The RAVE DR5 version (Kunder et al. 2017) contains data on 457588 stars; there is an overlap with the TGAS catalogue for about half of these stars.

In this paper we use the data set from Hunt et al. (2016), where the common stars from the TGAS and RAVE DR5 catalogues were studied. There are trigonometric parallaxes 
and proper motions from the TGAS catalogue and radial velocities from the RAVE DR5 catalogue for 216201 stars in this list. The stars with a relative distance error of more than $10 \%$ were excluded when the sample was produced. Hunt et al. (2016) used both photometric distance estimates from the RAVE catalogue and trigonometric parallaxes from the TGAS catalogue. In this paper we calculate all distances to the stars using their trigonometric parallaxes.

\section{METHODS}

\section{Model Galactic Gravitational Potential}

The expressions for the potentials are considered in a cylindrical coordinate system $(R, \psi, z)$ with the coordinate origin at the Galactic center. In a rectangular coordinate system $(x, y, z)$ with the coordinate origin at the Galactic center the distance to a star (spherical radius) will be $r^{2}=x^{2}+y^{2}+z^{2}=R^{2}+z^{2}$.

The equations of motion for a test particle in an axisymmetric gravitational potential $\Phi$ can be derived (see Appendix A to Irrgang et al. (2013)) from the Lagrangian $£$ of the system:

$$
£(R, z, \dot{R}, \dot{\psi}, \dot{z})=0.5\left(\dot{R}^{2}+(R \dot{\psi})^{2}+\dot{z}^{2}\right)-\Phi(R, z) .
$$

Introducing the canonical momenta

$$
p_{R}=\partial £ / \partial \dot{R}=\dot{R}, \quad p_{\psi}=\partial £ / \partial \dot{\phi}=R^{2} \dot{\psi}, \quad p_{z}=\partial £ / \partial \dot{z}=\dot{z}
$$

we will obtain the Lagrange equations as a system of six first-order differential equations:

$$
\begin{aligned}
& \dot{R}=p_{R}, \\
& \dot{\psi}=p_{\psi} / R^{2}, \\
& \dot{z}=p_{z} \\
& \dot{p_{R}}=-\partial \Phi(R, z) / \partial R+p_{\psi}^{2} / R^{3}, \\
& \dot{p_{\psi}}=0 \\
& \dot{p_{z}}=-\partial \Phi(R, z) / \partial z .
\end{aligned}
$$

The fourth-order Runge-Kutta algorithm was used to integrate Eqs. (3).

In this paper we use a three-component model Galactic gravitational potential:

$$
\Phi=\Phi_{b}+\Phi_{d}+\Phi_{h},
$$

where the subscripts denote the bulge, disk, and halo, respectively.

In accordance with the convention adopted in Allen and Santillán (1991), we express the gravitational potential in units of $100 \mathrm{~km}^{2} \mathrm{~s}^{-2}$, the distances in $\mathrm{kpc}$, and the masses in units of the Galactic mass $M_{\text {gal }}=2.325 \times 10^{7} M_{\odot}$, corresponding to the gravitational constant $G=1$.

The bulge, $\Phi_{b}(r)$, and disk, $\Phi_{d}(r(R, z))$, potentials are represented by the expressions from Miyamoto and Nagai (1975):

$$
\begin{gathered}
\Phi_{b}(r)=-\frac{M_{b}}{\left(r^{2}+b_{b}^{2}\right)^{1 / 2}}, \\
\Phi_{d}(R, z)=-\frac{M_{d}}{\left\{R^{2}+\left[a_{d}+\left(z^{2}+b_{d}^{2}\right)^{1 / 2}\right]^{2}\right\}^{1 / 2}},
\end{gathered}
$$


Table 1: Parameters of the model Galactic potential

\begin{tabular}{|r|r|}
\hline$M_{b}$ & $386 \mathrm{M}_{g a l}$ \\
\hline$M_{d}$ & $3092 \mathrm{M}_{g a l}$ \\
\hline$M_{h}$ & $452 \mathrm{M}_{\text {gal }}$ \\
\hline$b_{b}$ & $0.2487 \mathrm{kpc}$ \\
\hline$a_{d}$ & $3.67 \mathrm{kpc}$ \\
\hline$b_{d}$ & $0.3049 \mathrm{kpc}$ \\
\hline$a_{h}$ & $1.52 \mathrm{kpc}$ \\
\hline
\end{tabular}

where $M_{b}$ and $M_{d}$ are the masses of the components, $b_{b}, a_{d}$, and $b_{d}$ are the scale lengths of the components in kpc.

The expression for the halo potential was derived by Irrgang et al. (2013) based on the expression for the halo mass from Allen and Martos (1986):

$$
m_{h}(<r)=\left\{\begin{array}{ll}
\frac{M_{h}\left(r / a_{h}\right)^{\gamma}}{1+\left(r / a_{h}\right)^{\gamma-1}}, & \text { if } r \leq \Lambda \\
\frac{M_{h}\left(\Lambda / a_{h}\right)^{\gamma}}{1+\left(\Lambda / a_{h}\right)^{\gamma-1}}=\text { const, } & \text { if } r>\Lambda
\end{array}\right\}
$$

It slightly differs from that given in Allen and Santillán (1991) and is

$$
\Phi_{h}(r)= \begin{cases}\frac{M_{h}}{a_{h}}\left(\frac{1}{(\gamma-1)} \ln \left(\frac{1+\left(r / a_{h}\right)^{\gamma-1}}{1+\left(\Lambda / a_{h}\right)^{\gamma-1}}\right)-\frac{\left(\Lambda / a_{h}\right)^{\gamma-1}}{1+\left(\Lambda / a_{h}\right)^{\gamma-1}}\right), & \text { if } r \leq \Lambda \\ -\frac{M_{h}}{r} \frac{\left(\Lambda / a_{h}\right)^{\gamma}}{1+\left(\Lambda / a_{h}\right)^{\gamma-1}}, & \text { if } r>\Lambda,\end{cases}
$$

where $M_{h}$ is the mass, $a_{h}$ is the scale length, the Galactocentric distance is $\Lambda=200 \mathrm{kpc}$, and the dimensionless coefficient $\gamma=2.0$.

The model parameters $M_{b}, M_{d}, M_{h}, b_{b}, a_{d}, b_{d}$, and $a_{h}$ were taken from Bajkova and Bobylev (2016), where they were refined based on a large set of present-day observational data. Their values are given in Table 1.

The circular rotation velocity of the Galaxy at the adopted Galactocentric distance of the Sun $R_{0}=8.3 \mathrm{kpc}$ is $244 \mathrm{~km} \mathrm{~s}^{-1}$ (Bajkova and Bobylev 2016). The peculiar velocity components of the Sun relative to the local standard of rest were taken to be $\left(U_{\odot}, V_{\odot}, W_{\odot}\right)_{L S R}=(10,11,7) \mathrm{km} \mathrm{s}^{-1}$ based on the results from Bobylev and Bajkova (2014a) in agreement with the results from Schönrich et al. (2010). We take into account the Sun's height above the Galactic plane $Z_{\odot}=16$ pc (Bobylev and Bajkova 2016). We neglect the star-Sun gravitational interaction.

In the case where the spiral density wave is taken into account (Lin and Shu 1964; Lin et al. 1969), the following term (Fernandez et al. 2008) is added to the right-hand side of 

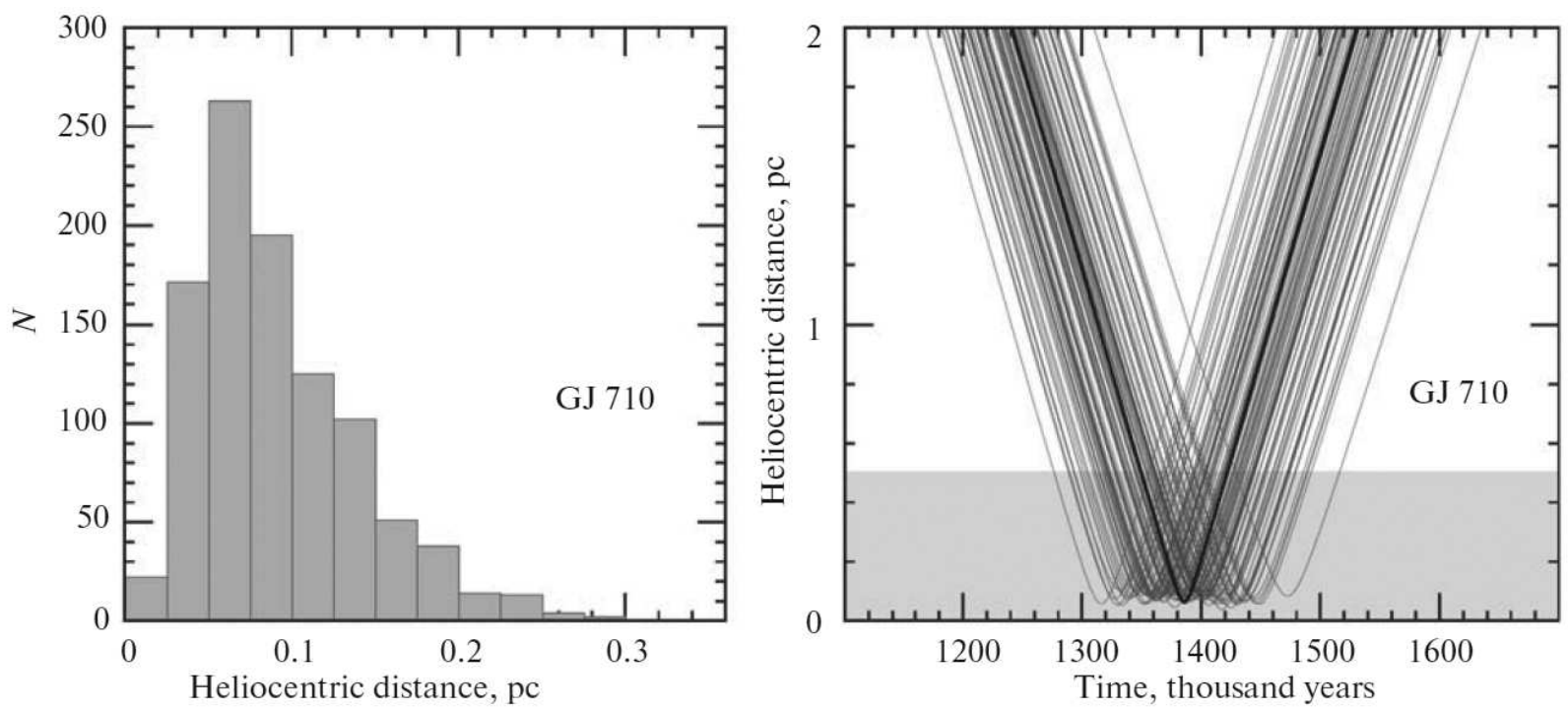

Figure 1: (a) Histogram of the distribution of model minimum distances $d_{m}$ obtained for the star GJ 710 by the Monte Carlo method for 1000 trajectories; (b) the thick line indicates the trajectory of GJ 710 relative to the Sun constructed with the parameters from Table 2 and 100 model trajectories obtained by the Monte Carlo method, the shading indicates the boundaries of the Oort cloud.

Eq. (2):

$$
\Phi_{s p}(R, \theta, t)=A \cos \left[m\left(\Omega_{p} t-\theta\right)+\chi(R)\right] .
$$

Here,

$$
A=\frac{\left(R_{0} \Omega_{0}\right)^{2} f_{r 0} \tan i}{m}, \quad \chi(R)=-\frac{m}{\tan i} \ln \left(\frac{R}{R_{0}}\right)+\chi_{\odot},
$$

where $A$ is the amplitude of the spiral potential, $f_{r 0}$ is the ratio between the radial component of the force due to the spiral arms and that due to the general Galactic field, $\Omega_{p}$ is the angular velocity of the spiral pattern, $m$ is the number of spiral arms, $i$ is the pitch angle of the arms ( $i<0$ for a wound pattern), $\chi$ is the radial phase of the spiral wave (the arm center then corresponds to $\chi=0^{\circ}$ ), and $\chi_{\odot}$ is the radial phase of the Sun in the spiral wave.

The following spiral wave parameters were taken as a first approximation:

$$
m=4, \quad i=-13^{\circ}, \quad f_{r 0}=0.05, \quad \chi_{\odot}=-140^{\circ}, \quad \Omega_{p}=20 \mathrm{~km} \mathrm{~s}^{-1} \mathrm{kpc}^{-1}
$$

We used this set of parameters in Bobylev and Bajkova (2014b), where a broad overview of the parameter selection problem is given. If necessary, some of them, in particular, $\chi_{\odot}$ and $\Omega_{p}$, can be varied.

\section{Monte Carlo Simulations}

In accordance with the method of Monte Carlo simulations, for each object we calculate a set of orbits by taking into account the random errors in the input data. For each star we calculate the parameter of the encounter between the stellar and solar orbits $d(t)=$ $\sqrt{\Delta x^{2}(t)+\Delta y^{2}(t)+\Delta z^{2}(t)}$. The closest encounter is characterized by two parameters, $d_{m}$ and $t_{m}$. The errors of the stellar parameters are assumed to be distributed as a normal law 


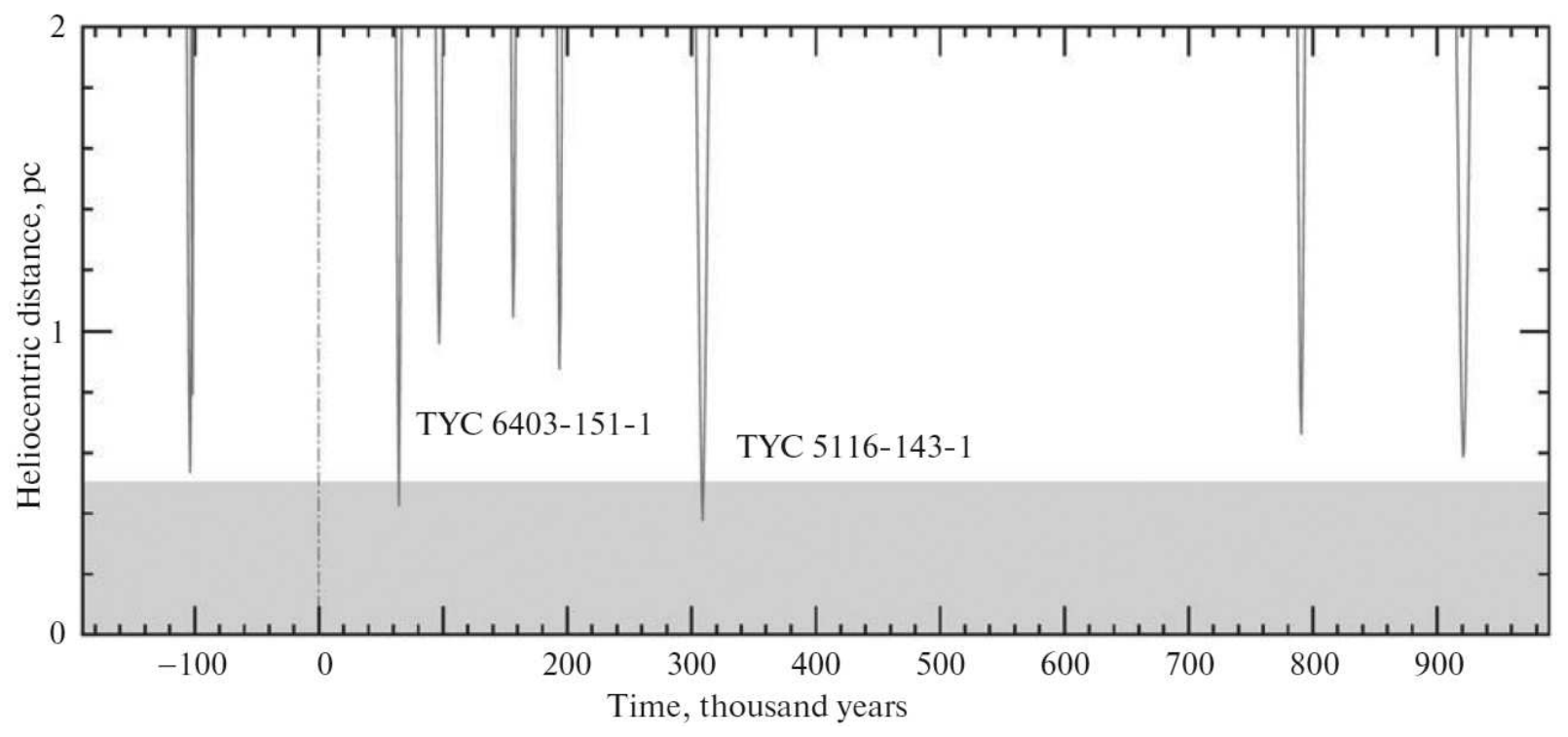

Figure 2: Trajectories of the nine stars from Table 3, the dash-dotted vertical line marks the current time, the shading indicates the boundaries of the Oort cloud.

with a dispersion $\sigma$. The errors are added to the stellar equatorial coordinates, proper motion components, parallaxes, and radial velocities.

\section{RESULTS AND DISCUSSION}

For each of the 216201 stars we constructed its orbit relative to the Sun in the time interval from -15 to $+15 \mathrm{Myr}$. From the entire list we selected the stars with an encounter parameter $d_{m}<1$ pc. We divided them into two samples, depending on the quality of the measured initial velocities and positions.

Sample 1 includes the stars for which the relative errors of the measured initial velocities and positions do not exceed $10 \%$ and the measurement error of the radial velocity $<15 \mathrm{~km}$ $\mathrm{s}^{-1}$. The parameters of the stars from sample 1 are given in Table 2, where columns 1-9 give, respectively, the ordinal star number, the Tycho identification number (the Hipparcos number is also provided, if available), the stellar equatorial coordinates $\alpha$ and $\delta$, the proper motion components $\mu_{\alpha} \cos \delta$, and $\mu_{\delta}$ with their measurement errors, the trigonometric parallax with its measurement error, the radial velocity with its measurement error, the signal-to-noise $(\mathrm{S} / \mathrm{N})$ ratio of the spectrum when determining the radial velocity copied from column 12 of the RAVE5 catalogue, and the encounter parameters $d_{m}$ and $t_{m}$ we found.

Many stars with huge radial velocities, which is most likely due to the erroneous measurements in the RAVE catalogue, enter into the sample. We collected them into separate Table 3. As can be seen, the $\mathrm{S} / \mathrm{N}$ ratios for the stars from Table 2 exceed those for the stars from Table 3 by an order of magnitude.

Several radial velocity determinations are given for some of the stars in the RAVE5 catalogue. These include, for example, TYC 5116-143-1, TYC 7567-304-1, TYC 5302-849-1, TYC 9163-286-1 or TYC 7978-659-1. The radial velocities usually differ by an order of magnitude. The encounter parameters calculated for several known radial velocity measurements are given in Bailer-Jones (2015) in such cases for each star. In contrast, we give only one 
Table 2: Data on the stars of sample 1

\begin{tabular}{|l|r|r|r|r|r|r|r|}
\hline $\begin{array}{l}\text { TYC } \\
\text { alternative } \\
\text { name }\end{array}$ & $\begin{array}{r}\alpha_{J 2000} \\
\delta_{J 2000} \\
\mathrm{deg}\end{array}$ & $\begin{array}{r}\mu_{\alpha} \cos \delta \\
\mu_{\delta} \\
\mathrm{mas} \mathrm{yr}\end{array}$ & $\pi$ & $V_{r}$ & $\mathrm{~S} / \mathrm{N}$ & $d_{m}$ & $\begin{array}{r}t_{m} \\
\text { mas }\end{array}$ \\
\hline $5102-100-1$ & 274.961836 & $-0.468 \pm 0.130$ & 52.35 & -13.8 & - & 0.063 & 1385 \\
GJ 710 & -01.938613 & $-0.176 \pm 0.090$ & \pm 0.27 & \pm 0.3 & & \pm 0.044 & \pm 52 \\
\hline $8088-631-1$ & 87.580551 & $0.353 \pm 0.670$ & 4.99 & 71.5 & 64.2 & 0.37 & -2792 \\
& -45.642797 & $0.037 \pm 0.940$ & \pm 0.22 & \pm 1.0 & & \pm 1.18 & \pm 66 \\
\hline $6528-980-1$ & 107.001787 & $0.221 \pm 0.605$ & 4.20 & 25.9 & 25.4 & 0.86 & -9115 \\
& -25.871894 & $0.625 \pm 0.599$ & \pm 0.23 & \pm 4.1 & & \pm 5.60 & \pm 35 \\
\hline
\end{tabular}

value at which the closest encounter is obtained.

Many of the stars from sample 2 have huge (more than $600 \mathrm{~km} \mathrm{~s}^{-1}$ ) space velocities. By this parameter they can be attributed to hypervelocity stars that are capable of escaping from the Galactic attractive field. The escape velocity at the Galactocentric distance of the Sun slightly depends on the model gravitational potential and is $\sim 550 \mathrm{~km} \mathrm{~s}^{-1}$ (see, e.g., Bajkova and Bobylev 2016). The following fact forces us to doubt that such high velocities are realistic. According to the well-known Kleiber theorem (Agekyan et al. 1962), the mean tangential, $V_{t}$, and radial, $V_{r}$, velocities are related by the relation $\left|\bar{V}_{t}\right|=0.5 \pi\left|\bar{V}_{r}\right|$. Although this relation is valid in the statistical sense, it does not hold at all in our case, because for all stars from sample $2\left|V_{t}\right|=4.74 r|\mu|<100 \mathrm{~km} \mathrm{~s}^{-1}$, where $\mu=\sqrt{\mu_{\alpha}^{2} \cos \delta+\mu_{\delta}^{2}}$.

Note that the flags $c_{1}-c_{20}$ describing the morphology of the spectra are specified in the RAVE catalogues. According to these characteristics, all of the detected stars with radial velocities $\left|V_{r}\right|>300 \mathrm{~km} \mathrm{~s}^{-1}$ have very low signal-to-noise ratios, and the spectra for all these stars are either with problems in their continuum $\left(c_{1,2,3}=\right.$ "c") or peculiar $\left(c_{1,2,3}=\right.$ "p"). This leads us to conclude that the radial velocities of such stars have been measured very poorly.

Note the star TYC 4888-146-1 (absent in our tables), for which four radial velocities found from four good $\left(c_{1,2,3}=\right.$ "n", the spectrum of a normal star) spectra taken at different epochs are given in the RAVE5 catalogue. All four values are close to $V_{r}=-15 \mathrm{~km} \mathrm{~s}^{-1}$, and one value $\left(V_{r}=1897 \mathrm{~km} \mathrm{~s}^{-1}\right)$ was found from a spectrum with problems in its continuum $\left(c_{1,2,3}=\right.$ "c"). All of this reinforces our attitude to the stars with huge radial velocities from the RAVE5 catalogue as problem ones.

The measurement error of the radial velocity $\sigma_{V_{r}}$ is unknown for several stars. In such cases, we adopted $\sigma_{V_{r}}= \pm 30 \mathrm{~km} \mathrm{~s}^{-1}$ for them when estimating the errors of the encounter parameters $d_{m}$ and $t_{m}$ by the Monte Carlo method.

Figure 1 gives a histogram of the distribution of model minimum distances $d_{m}$ obtained for the star GJ 710 by the Monte Carlo method for 1000 trajectories, 100 model trajectories of GJ 710 relative to the Sun.

Figure 2 gives the trajectories of the encounter of the nine stars from Table 3 with the solar orbit. All these trajectories resemble thin vertical lines attributable to large flyby velocities. Therefore, the impact of these stars on Oort cloud comets can only be very brief with minor consequences.

Bailer-Jones (2015) analyzed a large sample of stars with the radial velocities from the RAVE4 catalogue for close encounters. For example, for the star TYC 5116-143-1 (HIP 
Table 3: Data on the stars of sample 2

\begin{tabular}{|c|c|c|c|c|c|c|c|}
\hline $\begin{array}{l}\text { TYC } \\
\text { HIP } \\
(\mathrm{Sp})\end{array}$ & $\begin{array}{r}\alpha_{J 2000} \\
\delta_{J 2000} \\
\operatorname{deg}\end{array}$ & $\begin{array}{c}\mu_{\alpha} \cos \delta \\
\mu_{\delta} \\
\operatorname{mas} \mathrm{yr}^{-1}\end{array}$ & mas & $\mathrm{km} \mathrm{s}^{-1}$ & $\mathrm{~S} / \mathrm{N}$ & $d_{m}$ & $\begin{array}{r}t_{m} \\
\text { thousand } \\
\text { years }\end{array}$ \\
\hline $5116-143-1$ & 278.484540 & $-2.289 \pm 0.069$ & 8.92 & -364 & 1.2 & 0.38 & 308 \\
\hline $\begin{array}{l}\text { HIP } 91012 \\
(\mathrm{~F} 3 \mathrm{IV} / \mathrm{V})\end{array}$ & -2.820646 & $-0.005 \pm 0.052$ & \pm 0.31 & \pm 22 & & \pm 0.03 & \pm 28 \\
\hline $6403-151-1$ & $\begin{array}{r}354.359880 \\
-16.826037\end{array}$ & $\begin{array}{l}-14.114 \pm 1.840 \\
-21.315 \pm 0.891\end{array}$ & $\begin{array}{l}18.54 \\
\pm 0.56\end{array}$ & $\begin{array}{l}-851 \\
\pm 226\end{array}$ & 1.5 & $\begin{array}{r}0.42 \\
\pm 0.15\end{array}$ & $\begin{array}{r}64 \\
\pm 20\end{array}$ \\
\hline $6622-652-1$ & $\begin{array}{r}153.225519 \\
-25.002547\end{array}$ & $\begin{aligned}-14.047 & \pm 2.788 \\
3.054 & \pm 0.718\end{aligned}$ & $\begin{array}{r}13.14 \\
\pm 0.69\end{array}$ & $\begin{array}{l}741 \\
\pm 13\end{array}$ & 1.3 & $\begin{array}{r}0.53 \\
\pm 0.06\end{array}$ & $\begin{array}{r}-103 \\
\pm 8\end{array}$ \\
\hline $5508-848-1$ & $\begin{array}{r}170.955510 \\
-8.984829\end{array}$ & $\begin{aligned} 0.312 & \pm 2.297 \\
-0.244 & \pm 0.800\end{aligned}$ & $\begin{array}{r}3.24 \\
\pm 0.74\end{array}$ & $\begin{array}{r}-336 \\
\pm 8\end{array}$ & 1.9 & $\begin{array}{r}0.59 \\
\pm 0.75\end{array}$ & $\begin{array}{r}920 \\
\pm 25\end{array}$ \\
\hline $8923-1577-1$ & $\begin{array}{r}117.117101 \\
-66.890918\end{array}$ & $\begin{array}{r}0.164 \pm 0.884 \\
-0.278 \pm 0.703\end{array}$ & $\begin{array}{r}2.06 \\
\pm 0.34\end{array}$ & $\begin{array}{r}-614 \\
\pm 66\end{array}$ & 1.2 & $\begin{array}{r}0.66 \\
\pm 2.00\end{array}$ & $\begin{array}{r}790 \\
\pm 35\end{array}$ \\
\hline $8822-592-1$ & $\begin{array}{r}336.227642 \\
-54.123260\end{array}$ & $\begin{array}{r}-5.461 \pm 0.305 \\
3.249 \pm 0.578\end{array}$ & $\begin{array}{r}5.03 \\
\pm 0.25\end{array}$ & $\begin{array}{r}1969 \\
\pm 30\end{array}$ & 0.7 & $\begin{array}{r}0.79 \\
\pm 7\end{array}$ & $\begin{array}{r}-101 \\
\pm 8\end{array}$ \\
\hline $\begin{array}{l}9524-1668-1 \\
(\mathrm{~F} 2 \mathrm{~V})\end{array}$ & $\begin{array}{r}325.449945 \\
-82.958804\end{array}$ & $\begin{array}{l}3.626 \pm 0.621 \\
4.032 \pm 0.563\end{array}$ & $\begin{array}{r}5.84 \\
\pm 0.38\end{array}$ & -887 & 1.9 & $\begin{array}{r}0.87 \\
\pm 0.11\end{array}$ & $\begin{array}{r}193 \\
\pm 5\end{array}$ \\
\hline $7567-304-1$ & $\begin{array}{r}48.605192 \\
-44.689565\end{array}$ & $\begin{array}{r}-20.624 \pm 0.613 \\
28.482 \pm 1.114\end{array}$ & $\begin{array}{l}16.67 \\
\pm 0.23\end{array}$ & $\begin{array}{r}-625 \\
\pm 25\end{array}$ & 1.7 & $\begin{array}{r}0.96 \\
\pm 0.10\end{array}$ & $\begin{array}{r}96 \\
\pm 5\end{array}$ \\
\hline 9339-404-1 & $\begin{array}{r}358.159161 \\
-69.790350\end{array}$ & $\begin{array}{l}9.592 \pm 0.884 \\
0.293 \pm 0.633\end{array}$ & $\begin{array}{r}6.91 \\
\pm 0.25\end{array}$ & $\begin{array}{r}-929 \\
\pm 29\end{array}$ & 0.9 & $\begin{array}{r}1.04 \\
\pm 0.10\end{array}$ & $\begin{array}{r}155 \\
\pm 9\end{array}$ \\
\hline
\end{tabular}

91012) (Table 3) he found $d_{m}=0.48 \mathrm{pc}$ and $t_{m}=302$ thousand years when using the radial velocity $V_{r}=-364 \mathrm{~km} \mathrm{~s}^{-1}$ (as we did). To integrate the stellar orbits, Bailer-Jones (2015) used a model Galactic gravitational potential different from ours. In spite of this, we can conclude that we have results very close to those obtained by other authors when using the same observational data. As can be seen from Table 3, interesting encounter parameters, with small random errors, were obtained for this star. However, the RAVE5 catalogue provides another radial velocity for it, $V_{r}=-36.5 \pm 18.6 \mathrm{~km} \mathrm{~s}^{-1}$, obtained from a different but, just as in the former case, poor spectrum. In addition, according to the measurements by Nordström et al. (2004), $V_{r}=-16.8 \pm 0.4 \mathrm{~km} \mathrm{~s}^{-1}$. We found that with such a radial velocity the encounter of TYC 5116-143-1 (HIP 91012) would not be close $\left(d_{m}>5 \mathrm{pc}\right)$.

The star GJ 710 is known quite well. For example, $T_{\text {eff }}=4109 \mathrm{~K}$ and $\log (g)=4.91 \mathrm{~cm}$ $\mathrm{s}^{-2}$ for it (Franchini et al. 2014), i.e., this is an orange dwarf with a mass of $\sim 0.6 M_{\odot}$. Since there is no radial velocity for it in the RAVE catalogues, we took its previously known value from the catalogue by Gontcharov (2006). Using the new trigonometric parallax and proper motions from the Gaia DR1 catalogue, we found the encounter parameters $d_{m}=0.063 \pm 0.044$ pc and $t_{m}=1385 \pm 52$ thousand years, which are in excellent agreement with the estimates obtained by Berski and Dybczyński (2016) using the same data and a similar technique for calculating the Galactic stellar orbits, $d_{m}=0.065 \pm 0.030 \mathrm{pc}$ and $t_{m}=1350 \pm 50$ thousand years.

The following parameters are given in the RAVE5 catalogue for the other stars from Table 2: $T_{\text {eff }}=5940 \mathrm{~K}, \log (g)=4.08 \mathrm{~cm} \mathrm{~s}^{-2}$ for TYC 8088-631-1 and $T_{\text {eff }}=4750 \mathrm{~K}$, 
$\log (g)=4.000 \mathrm{~cm} \mathrm{~s}^{-2}$ for TYC 6528-980-1. They show that these stars are dwarfs.

Previously, Dybczyński (2006) and Jimeénez-Torres et al. (2011) performed numerical simulations of the evolution of comet orbits using the penetration of a star like GJ 710 with a mass of $0.6 M_{\odot}$ and $d_{m}=0.3 \mathrm{pc}$ into the Oort cloud as an example and found a small stream of comets toward the major planets from the impact of a model star that is difficult to separate from the stream of comets caused by a Galactic tide. However, using a closer encounter parameter, $d_{m}=0.065$ pc, Berski and Dybczyński (2016) showed that a noticeable stream with a density of about ten comets per year with a duration of 2-4 Myr could emerge.

\section{CONCLUSIONS}

We searched for the stars that encountered or would encounter with the Solar system closer than 1 pc. For this purpose, we took more than 216000 stars with the measured proper motions and trigonometric parallaxes from the Gaia DR1 catalogue and their radial velocities from the RAVE5 catalogue. The orbits were integrated over the time interval from -15 to +15 Myr using a model Galactic gravitational potential that includes an axisymmetric part (bulge, disk, and halo)with the addition of a nonaxisymmetric component that allows for the influence of the Galactic spiral density wave.

We found the stars for which encounters with the Solar system closer than 1 pc are possible. For the bulk of this list such an analysis has been made for the first time. We divided all of the stars found into two samples.

Sample 1 contains the stars with small errors of the input data and low radial velocities. The star GJ 710, for which the minimum distance is $d_{m}=0.063 \pm 0.044 \mathrm{pc}$ at time $t_{m}=$ $1385 \pm 52$ thousand years, is the record-holder in this sample. This confirms the estimates that have recently been obtained for GJ 710 from similar data by Berski and Dybczyński (2016). The first sample includes two more stars, TYC 8088-631-1 and TYC 6528-980-1, with

$d_{m}<1$ pc, which, however, are estimated with large errors. For example, $d_{m}=0.37 \pm 1.18 \mathrm{pc}$ and $t_{m}=-2792 \pm 66$ thousand years were found for TYC 8088-631-1.

The remaining stars enter into sample 2. They are characterized by unrealistically large space velocities and their random errors. This is because poor quality spectra were used for these stars in the RAVE catalogues. Therefore, the results obtained from the stars of sample 2 are much less trustworthy than the previous ones.

\section{ACKNOWLEDGMENTS}

We are grateful to the referee for the useful remarks that contributed to an improvement of the paper. We are thankful to J. Hunt for the provided data. This work was supported by the Basic Research Program P-7 of the Presidium of the Russian Academy of Sciences, the "Transitional and Explosive Processes in Astrophysics" Subprogram.

\section{REFERENCES}

1. T.A. Agekyan, B.A. Vorontsov-Vel'yaminov, V.G. Gorbatskii, A.N. Deich, V.A. Krat, O.A. Melnikov, and V.V. Sobolev, Course of Astrophysics and Stellar Astronomy (Fizmatgiz, Moscow, 1962) [in Russian].

2. C. Allen and M.A. Martos, Rev. Mex. Astron. Astrofis. 13, 137 (1986).

3. C. Allen and A. Santillán, Rev. Mex. Astron. Astrofis. 22, 255 (1991).

4. E. Anderson and C. Francis, Astron. Lett. 38, 331 (2012).

5. C.A.L. Bailer-Jones, Astron. Astrophys. 575, 35 (2015). 
6. A.T. Bajkova and V.V. Bobylev, Astron. Lett. 42, 567 (2016).

7. F. Berski and P.A. Dybczyński, Astron. Astrophys. 595, L10 (2016).

8. V.V. Bobylev, Astron. Lett. 36, 220 (2010a).

9. V.V. Bobylev, Astron. Lett. 36, 816 (2010b).

10. V. Bobylev and A. Bajkova, Mon. Not. R. Astron. Soc. 441, 142 (2014a).

11. V.V. Bobylev and A.T. Bajkova, Astron. Lett. 40, 352 (2014b).

12. V.V. Bobylev and A.T. Bajkova, Astron. Lett. 42, 1 (2016).

13. A.G.A. Brown, A. Vallenari, T. Prusti, J. de Bruijne, F. Mignard, R. Drimmel, et al. (GAIA Collab.), Astron. Astrophys. 595, 2 (2016).

14. P.A. Dybczyński, Astron. Astrophys. 396, 283 (2002).

15. P.A. Dybczyński, Astron. Astrophys. 441, 783 (2005).

16. P.A. Dybczyński, Astron. Astrophys. 449, 1233 (2006).

17. P.A. Dybczyński and M. Królikowska, Mon. Not. R. Astron. Soc. 416, 51 (2011).

18. P.A. Dybczyński and F. Berski, Mon. Not. R. Astron. Soc. 449, 2459 (2015).

19. V.V. Emel'yanenko, D. J. Asher, and M. E. Bailey, Mon. Not. R. Astron. Soc. 381, 779 (2007).

20. F. Feng and C.A.L. Bailer-Jones, Mon. Not. R. Astron. Soc. 454, 3267 (2015).

21. D. Fernandez, F. Figueras, and J. Torra, Astron. Astrophys. 480, 735 (2008).

22. M. Franchini, C. Morossi, P. di Marcantonio, M.L. Malagnini, and M. Chavez, Mon. Not.

R. Astron. Soc. 442, 220 (2014).

23. J. Garcia-Sánchez, R.A. Preston, D. L. Jones, P.R. Weissman, J.-F. Jean-Francois, D.W. Latham, and R.P. Stefanik, Astron. J. 117, 1042 (1999).

24. J. Garcia-Sánchez, P.R. Weissman, R.A. Preston, D.L. Jones, J.-F. Lestrade, D.W. Latham, R.P. Stefanik, and J.M. Paredes, Astron. Astrophys. 379, 634 (2001).

25. G. A. Gontcharov, Astron. Lett. 32, 759 (2006).

26. J. G. Hills, Astron. J. 86, 1730 (1981).

27. The Hipparcos and Tycho Catalogues, ESA SP-1200 (1997).

28. E. Hog, C. Fabricius, V.V. Makarov, U. Bastian, P. Schwekendiek, A. Wicenec, S. Urban, T. Corbin, and G. Wycoff, Astron. Astrophys. 355, L27 (2000).

29. J.A.S. Hunt, J. Bovy, and R.G. Carlberg, Astrophys. J. 832, L25 (2016).

30. A. Irrgang, B. Wilcox, E. Tucker, and L. Schiefelbein, Astron. Astrophys. 549, 137 (2013).

31. J.J. Jiménez-Torres, B. Pichardo, G. Lake, and H. Throop, Mon. Not. R. Astron. Soc. 418, $1272(2011)$.

32. A. Kunder, G. Kordopatis, M. Steinmetz, T. Zwitter, P. McMillan, L. Casagrande, H. Enke, J. Wojno, et al., Astron. J. 153, 75 (2017).

33. G. Leto, M. Jakubik, T. Paulech, L. Neslušan, and P.A. Dybczyński, Mon. Not. R. Astron. Soc. 391, 1350 (2008).

34. C.C. Lin and F.H. Shu, Astrophys. J. 140, 646 (1964).

35. C.C. Lin, C. Yuan and F.H. Shu, Astrophys. J. 155, 721 (1969).

36. L. Lindegren, U. Lammers, U. Bastian, J. Hernandez, S. Klioner, D. Hobbs, A. Bombrun, D. Michalik, et al., Astron. Astrophys. 595, 4 (2016).

37. E.E. Mamajek, S.A. Barenfeld, V.D. Ivanov, A.Y. Kniazev, P. Väisänen, Y. Beletsky, and H.M.J. Boffin, Astrophys. J. Lett. 800, L17 (2015).

38. C. A. Martinez-Barbosa, L. Jýlková, S. Portegies Zwart, and A.G. A. Brown, Mon. Not. R. Astron. Soc. 464, 2290 (2017).

39. R. A. J. Matthews, R. Astron. Soc. Quart. J. 35, 1 (1994).

40. D. Michalik, L. Lindegren, and D. Hobbs, Astron. Astrophys. 574, 115 (2015).

41. M. Miyamoto and R. Nagai, Publ. Astron. Soc. Jpn. 27, 533 (1975).

42. A. A. Mülläri and V. V. Orlov, Earth, Moon, Planets 72, 19 (1996). 
43. B. Nordström, M. Mayor, J. Andersen, J. Holmberg, F. Pont, B. R. Jorgensen, E. H. Olsen, S. Udry, and N. Mowlavi, Astron. Astrophys. 418, 989 (2004).

44. J.H. Oort, Bull. Astron. Inst. Netherland 11 (408), 91 (1950).

45. T. Prusti, J.H. J. de Bruijne,A.G. A. Brown, A. Vallenari, C. Babusiaux, C. A. L. BailerJones, U. Bastian, M. Biermann, et al. (GAIA Collab.), Astron. Astrophys. 595, 1 (2016).

46. H. Rickman, M. Fouchard, C. Froeschlé, and G. B. Valsecchi, Sel. Mech. Dyn. Astron. 102, 111 (2008).

47. R. Schönrich, J. Binney, and W. Dehnen, Mon. Not. R. Astron. Soc. 403, 1829 (2010).

48. M. Steinmetz, T. Zwitter, A. Siebert, F. G. Watson, K. C. Freeman, U. Munari, R. Campbell, M. Williams, et al., Astron. J. 132, 1645 (2006).

49. J. T. Wickramasinghe and W. M. Napier, Mon. Not. R. Astron. Soc. 387, 153 (2008). 